Acta Zoológica Mexicana (n.s.) 23(1): 37-46 (2007)

\title{
ESPECIES DE ERETMOCERUS HALDEMAN (HYMENOPTERA: APHELINIDAE) - PARASITOIDES DE ALEUROTHRIXUS FLOCCOSUS (MASKELL) (HOMOPTERA: ALEYRODIDAE) DE MÉXICO, CON LA DESCRIPCIÓN DE UNA NUEVA ESPECIE
}

\author{
S. N. Myartseva y J. M. Coronado-Blanco \\ División de Estudios de Postgrado e Investigación, \\ UAM Agronomía y Ciencias, Universidad Autónoma de Tamaulipas, \\ Ciudad Victoria, 87149, Tamaulipas, MÉXICO \\ smyartse@uat.edu.mx; jmcoronado@uat.edu.mx \\ RESUMEN
}

Una nueva especie es descrita, Eretmocerus naranjae. Se incluye una clave de las seis especies de Eretmocerus, parasitoides de la mosquita blanca Aleurothrixus floccosus (Maskell) de México, así como la diagnosis, hospederos, distribución y material examinado de cuatro de las especies colectadas. Palabras Clave: Eretmocerus, Eretmocerus naranjae sp. n., Aleurothrixus floccosus.

\section{ABSTRACT}

A new species is described, Eretmocerus naranjae. A key to six species of Eretmocerus parasitoids of whitefly Aleurothrixus floccosus (Maskell) from Mexico, is given. Diagnosis, hosts, distribution and examined material for four collected species are given.

Key Words: Eretmocerus, Eretmocerus naranjae sp. n., Aleurothrixus floccosus.

\section{INTRODUCCIÓN}

El género Aleurothrixus está muy bien representado en el nuevo mundo, 15 de las 18 especies descritas son de origen americano incluyendo a $A$. floccosus (Maskell) conocida vulgarmente como "mosquita blanca lanuda de los cítricos". Esta especie es conocida a nivel mundial como una seria plaga que daña los cítricos; en la década del 60 invadió el sur de Europa, área mediterránea, Islas Canarias y otros sitios del viejo mundo. Rose \& De Bach (1994) publicaron una monografía sobre A. floccosus, resultado de más de 14 años de investigaciones sobre esta especie y sus enemigos naturales en California y otras regiones. Dichos autores estimaron que el papel de los enemigos naturales en la disminución de las poblaciones de mosquitas blancas y de las infestaciones en cítricos ha sido relevante.

Con frecuencia un complejo de himenópteros parasitoides ataca a $A$. floccosus incluyendo especies nativas o introducidas. La mayor parte de las especies del 
complejo pertenecen a los géneros Encarsia Foerster y Eretmocerus Haldeman. Por tanto, el estudio de los enemigos naturales de las mosquitas blancas tiene importancia económica para el desarrollo de su control biológico.

En México se conocían hasta el año 2000, sólo cuatro especies de afelínidos parasitoides de A. floccosus: dos especies del género Encarsia, una de Encarsiella Hayat y una de Eretmocerus (Myartseva \& Ruíz Cancino 2000; Noyes 2005). Posteriormente, Rose (2000) publica una monografía señalando 11 especies de Eretmocerus obtenidas de esta mosquita blanca en el continente americano; tres nuevas especies se describieron para diferentes estados de México.

El objetivo de este trabajo es el aporte al conocimiento de las especies parasitoides de la especie plaga Aleurothrixus floccosus en México, se describe una nueva especie de enemigo natural y se brinda una clave y la diagnosis de las especies parasitoides de esta plaga en el país.

\section{MATERIAL Y MÉTODOS}

Los especimenes de Eretmocerus fueron colectados, criados y preservados usando la metodología corriente de acuerdo a Noyes (1982). Asimismo para la identificación de las especies se utilizaron las claves de Rose (2000) y Hayat (1998) y se estudiaron las descripciones originales de las especies descritas por estos autores. En este artículo se incluye también a Eretmocerus paulistus Hempel y E. portoricensis Dozier, parasitoides de A. floccosus. Estas dos especies fueron registradas para México por De Santis (1979, 1989) pero las autoras de este trabajo no las han encontrado en Tamaulipas, siendo su opinión que posiblemente no se encuentren en México.

Los materiales de Eretmocerus de la Universidad Autónoma de Tamaulipas, fueron obtenidos de A. floccosus durante 1998-2006 en México, principalmente en localidades de Tamaulipas. En el presente artículo se anotan diagnosis, hospederos, distribución y una clave que incluye a las seis especies mexicanas de Eretmocerus que parasitan a la mosquita blanca lanuda de los cítricos. En la distribución, el asterisco representa un nuevo registro para el Estado.

El material tipo de la nueva especie Eretmocerus naranjae, será depositado en el Research Entomological Museum, University of California, Riverside, Estados Unidos de América (UCRC). El resto de los especimenes se encuentra depositado en el Museo de Insectos de la Unidad Académica Multidisciplinaria Agronomía y Ciencias, de la Universidad Autónoma de Tamaulipas, Ciudad Victoria, Tamaulipas, México.

\section{RESULTADOS}

A continuación se incluye la diagnosis de Eretmocerus y una clave de las seis especies que se desarrollan como parasitoides de A. floccosus en México; asimismo se brindan 
las diagnosis, hospederos, distribución y material examinado de las cuatro especies colectadas.

\section{Eretmocerus Haldeman}

Eretmocerus Haldeman, 1850: 111 (especie tipo Eretmocerus corni Haldeman, por monotipia). Ricinusa Risbec, 1951: 403 (especie tipo Ricinusa aleyrodiphaga Risbec, por designación original); sinonimizado por Ferriere, 1965: 170.

\section{Diagnosis.}

Hembra con antena de 5 segmentos; radícula/escapo cilíndricos, pedicelo, dos segmentos funiculares cortos aneliformes o triangulares, maza grande, cilíndrica o gradualmente ensanchada hacia la porción distal y espatulada o fusiforme, con sensilas longitudinales dispersas. Mandíbulas tridentadas o bidentadas y un borde romo corto. Tórulos antenales más cercanos al margen de la boca. Ojos pequeños. Ocelos usualmente dispuestos en triángulo obtuso. Lóbulo medio del mesoescudo con 4 o 6 setas largas. Escutelo con dos pares de setas largas. Propodeo usualmente con una proyección triangular medialmente en el margen posterior, sobre el margen anterior con una seta aplanada, con un ápice bífido cerca de cada espiráculo. Endofragma bien desarrollado. Ala anterior y longitud del fleco marginal variables en dimensiones. Vena marginal usualmente con 3 o 4 setas, vena submarginal con 2 o 3 setas. Disco con setas dispersas, con la línea calva cerrada posteriormente por unas pocas setas y tubérculos, celda basal con 1 a 3 setas. Ala posterior estrecha, longitud del fleco marginal variable. Patas largas y delgadas, fórmula tarsal 4-4-4, espolón de la tibia media usualmente cerca de $0.5 \mathrm{X}$ la longitud del basitarso medio. Metasoma de longitud variable pero usualmente más largo que la cabeza y el mesosoma juntos. Ovipositor ligeramente expuesto o no expuesto pero las valvas del ovipositor pueden estar fuertemente expuestas, algunas veces con el ápice curvado.

Macho con antena compuesta de radícula, escapo, pedicelo y maza (sin segmentos funiculares). Maza larga y curvada, presenta numerosas sensilas. Falobase de la genitalia ventralmente con dos prolongaciones distales en forma de varilla, digitus bien desarrollados, cada uno con dos dentículos.

Longitud del cuerpo usualmente de 0.4 a $0.8 \mathrm{~mm}$. Color del cuerpo predominantemente amarillo, con un mínimo de zonas de color pardo. Escultura finamente reticulada.

Hospederos. Aleyrodidae, raramente otros "homópteros".

Distribución. Casi cosmopolita.

\section{Clave para especies de Eretmocerus, parasitoides de Aleurothrixus floccosus en México (Hembras)}

1. Lóbulo medio del mesoescudo con 4 setas. Maza antenal 3.5 veces tan larga como ancha, 1.25 veces tan larga como el ovipositor y 1.05 veces tan larga como la tibia media; primer segmento funicular triangular, segundo segmento comprimido, subcuadrado (Fig. 5).......

5. paulistus Hempel

- Lóbulo medio del mesoescudo con 6 setas.

2. Primer segmento funicular en forma de triángulo rectángulo y base elongada, segundo segmento comprimido (Fig. 2) ..... 2. jimenezi Rose - Primer segmento funicular triangular sin base elongada, segundo segmento 
Myartseva \& Coronado-Blanco: Seis especies de parasitoides de la mosquita blanca

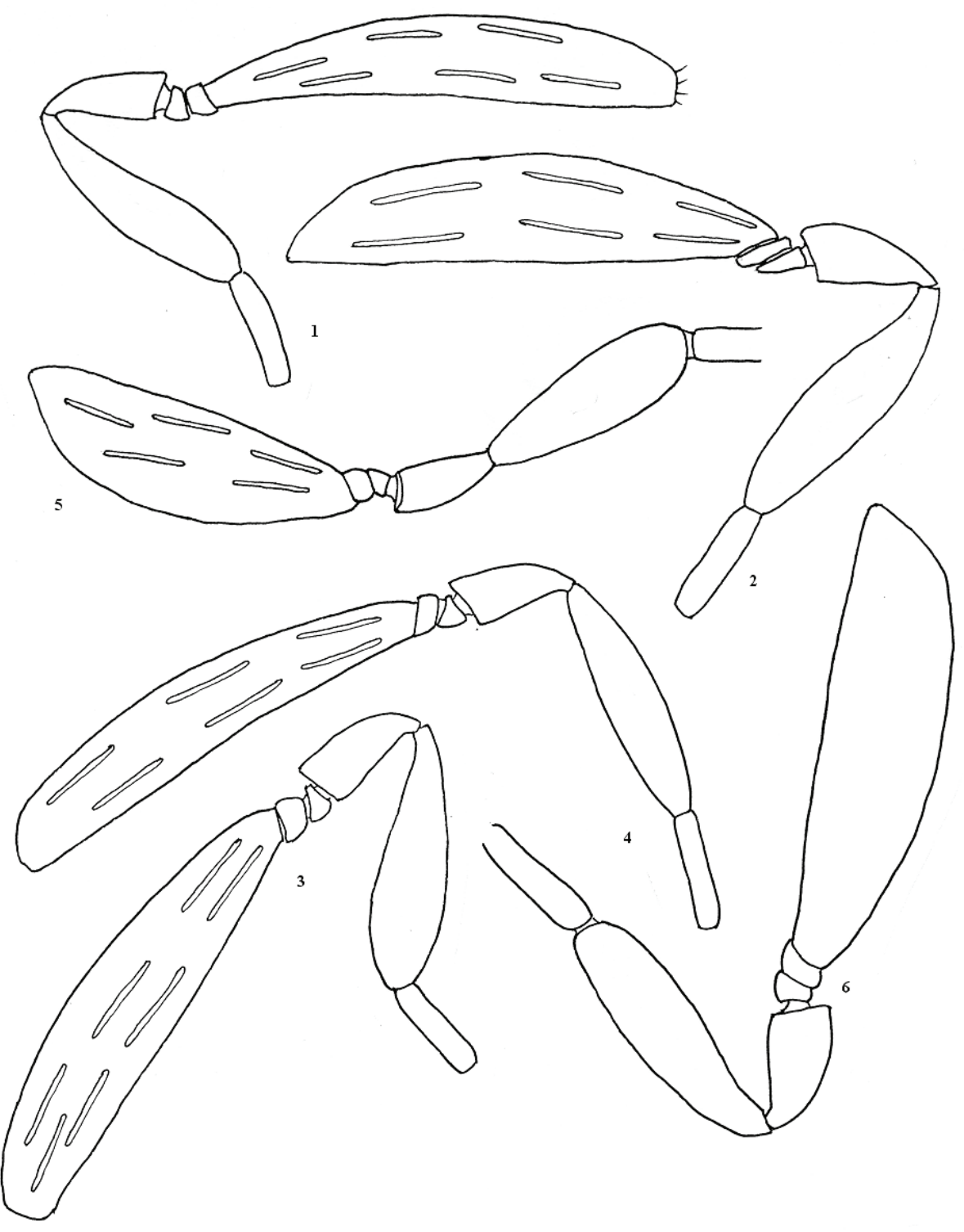

Figuras 1 - 6

Eretmocerus spp. Antenas de las hembras: 1- E. comperei Rose; 2- E. jimenezi Rose; 3- E. longiterebrus Rose; 4- E. naranjae sp. nov.; 5- E. paulistus Hempel (tomado de Rose, 2000); 6- E. portoricensis Dozier (tomado de Rose, 2000). 
subcuadrado a casi cuadrado o ligeramente más largo que ancho o más ancho que largo ... 6. portoricensis Dozier

3. Maza antenal hasta 3.8 veces tan larga como ancha (Fig. 6)

- Maza antenal más de 4 veces tan larga como ancha ................................................. 4

4. Ovipositor cuando mucho 1.30 veces tan largo como la maza y 1.16 veces tan largo como la tibia media, maza de 4.5 a 5.4 veces tan larga como ancha (Fig. 3)

.3 longiterebrus Rose

- Ovipositor cuando mucho 1.20 veces tan largo como la maza, longitud o anchura de la maza variable

5. Segundo segmento funicular subcuadrado, maza (Fig. 1) usualmente 5.5 veces tan larga como ancha o ligeramente más larga pero no más de 6 veces, ovipositor tan largo como la maza 1. comperei Rose - Segundo segmento funicular distintamente transversal, maza hasta 6.5 veces tan larga como ancha (Fig. 4), ovipositor 1.1 veces tan largo como la maza 4. naranjae sp. $n$.

\section{Sinopsis de las especies}

\section{Eretmocerus comperei Rose}

Eretmocerus comperei Rose, 2000: 16 -18, Figs. 13 -14.

Descrita de México, holotipo hembra en National Museum of Natural History, Washington, D. C., U.S.A. (USNM).

Hospederos: Aleurothrixus floccosus (Maskell), Siphoninus phillyreae (Haliday), Tetraleurodes sp.

Distribución. México: Baja California Sur, Colima, Guerrero, Nayarit, *Nuevo León, *San Luis Potosí, Sinaloa, *Tamaulipas.

Diagnosis. Maza antenal fusiforme (Fig. 1), siempre más de 5.5 veces pero menos de 6 veces tan larga como ancha, escapo cerca de 4.3 veces tan largo como ancho, pedicelo cerca de 2.4 veces tan largo como ancho, el primer segmento funicular en forma de triángulo y el segundo segmento subcuadrado; el lóbulo medio del mesoescudo con 6 setas, ala anterior cerca de 2.5 veces tan larga como ancha, longitud de la maza en relación con el ovipositor y la tibia media 1:1:1.05, respectivamente; longitud de la tibia media/basitarso cerca de 1:1.7.

Material examinado (obtenido de A. floccosus). México - Tamaulipas, Cd. Victoria, en Citrus limon, Citrus aurantifolia, 1 hembra, 9.II.1999, 2 hembras, 17.II.1999, 1 hembra, 1.V.1999, 1 hembra, 4.IV.2000, 2 hembras, 15.II.2003, ex Siphoninus phillyreae en Fraxinus spp., 1 hembra, 3.III.2006; Cd. Victoria, UAT, en Psidium guajava, 2 hembras, 25.XI.1999, 1 hembra, 3.II.2003; Miquihuana, ex Tetraleurodes sp. en arbusto, 1 hembra, 3.X.1998; Jaumave, en Citrus limon, 5 hembras, 4.X.1998; Jaumave, Paso Real, ex Tetraleurodes sp. en Quercus sp., 2 hembras, 31.III.2001; Cd. Mante, en Citrus aurantifolia, 1 hembras, 7.V.2004. San Luis Potosí, Santa María del Río, en Citrus limon, 1 hembra, 13.XI.1999. Nuevo León, Galeana, ex Tetraleurodes sp. en Mahonia trifoliata, 1 hembra, 7.VII.2000. Sinaloa, 15 km S Los Mochis, en Citrus spp., 1 hembra, 10.XI.2004 (S. Myartseva, col.).

Comentarios. Nuevo registro para los estados de Nuevo León, San Luis Potosí y Tamaulipas. Nuevos hospederos: Siphoninus phillyreae (Haliday) y Tetraleurodes sp. 
Myartseva \& Coronado-Blanco: Seis especies de parasitoides de la mosquita blanca

\section{Eretmocerus jimenezi Rose}

Eretmocerus jimenezi Rose, 2000: 26 -29, Figs. 3, 23 -24.

Descrita de México, hembra holotipo en USNM.

Hospedero. Aleurothrixus floccosus (Maskell).

Distribución. El Salvador; México - Colima, Guerrero, Michoacán, Nayarit, Nuevo León, San Luis Potosí, Sinaloa, *Tamaulipas, Veracruz.

Diagnosis. Maza antenal cerca de 4.3 veces tan larga como ancha (Fig. 2), escapo cerca de 3.8 veces tan largo como ancho, pedicelo cerca de 2.1 veces tan largo como ancho, primer segmento funicular en forma de un triángulo recto elongado, segundo segmento reducido, muy comprimido y en forma de 'luna creciente'; lóbulo medio del mesoescudo con 6 setas; ala anterior cerca de 2.5 veces tan larga como la anchura máxima del ala, fleco marginal cerca de 1/5 la anchura del disco, 9-11 setas entre la vena marginal y la línea calva; longitud de la espina de la tibia media/basitarso cerca de 1:1.6; longitud de la maza en relación con el ovipositor y la tibia media 1:1.2, 1:1.25, es decir, ovipositor más o menos de la longitud de la tibia media.

Material examinado (obtenido de A. floccosus). México - Tamaulipas, Alta Cima, en Psidium guajava, 7 hembras, 13.XI.1998; Gómez Farías, en Citrus spp., 2 hembras, 7.III.2006, 1 hembra, 17.III.2006; Cd. Mante, en Citrus spp., 1 hembra, 7.V.2004; Cd. Victoria, en Citrus spp., 1 hembra, 17.II.1999, 6 hembras, 27.X.1999, 3 hembras, 1.V.1999, 4 hembras, 9.V.1999, 2 hembras, 30.III.2003, ex Tetraleurodes sp. en Leucaena pulverulenta, 2 hembras, 17.I.2000; Güémez, Aleyrodidae en Citrus spp., 1 hembra, 25.V.2005, 2 hembras, 30.IV.2005 (S. Varela, col.), 2 hembras, 10.V.2005 (S. Myartseva, col.). Nuevo León, 20 km S Monterrey, en Citrus spp., 2 hembras, 7.VI.2005 (S. Myartseva, E. Ruíz Cancino, cols.). Colima, Tecomán, ex Aleurothrixus floccosus en Citrus limon (mexicano), 5 hembras, 2-VI-2006 (S. Myartseva, col.). Comentarios. En México esta especie fue identificada previamente como Eretmocerus portoricensis Dozier (Myartseva et al., 2004; Ruíz Cancino et al., 2005; Myartseva y Ruíz Cancino, 2005). Nuevo registro para el Estado de Tamaulipas.

\section{Eretmocerus longiterebrus Rose}

Eretmocerus longiterebrus Rose, 2000: 39 - 41, Figs. 25 -26.

Descrita de México, hembra holotipo en USNM.

Hospederos: Aleurothrixus floccosus, Tetraleurodes sp.

Distribución. México: Chiapas, Coahuila, Colima, Jalisco, Michoacán, Morelos, Puebla, Querétaro, *San Luis Potosí, Sinaloa, Sonora, *Tamaulipas; *Estados Unidos de América (California).

Diagnosis. Maza antenal cerca de 4.5 veces tan larga como ancha (Fig. 3), escapo cerca de 4.2 veces tan largo como ancho, pedicelo cerca de 2.2 veces tan largo como ancho, primer segmento funicular triangular, segundo segmento subcuadrado y más ancho que el primero, lóbulo medio del mesoescudo con 6 setas; ala anterior cerca de 2.4 veces tan larga como ancha, fleco marginal cerca de 1/5 la anchura del ala; 12-15 setas entre la vena marginal y línea calva; longitud de la espina de la tibia media/basitarso cerca de 1.0:1.75; ovipositor fuertemente expuesto, su punta con frecuencia ligeramente curvada; su longitud cerca de $1.26 \times$ la longitud de la maza y $1.16 \times$ la longitud de la tibia media.

Material examinado: (obtenido de A. floccosus). México: Tamaulipas, Cd. Victoria, en Citrus limon, 4 hembras, 4.IV.2000, en Citrus aurantifolia, 2 hembras, 30.III.2003, Cañón del Novillo, ex Tetraleurodes sp. en arbusto, 1 hembra, 26.XI.1998; Cd. Mante, en Citrus spp., 1 hembra, 7.V.2004. Chiapas, Tapachula, ex Tetraleurodes sp. on Bauhinia variegata, 4 hembras, 
30.V.2005. San Luis Potosí, Santa María del Río, ex Aleurothrixus floccosus en Citrus limon, 2 hembras, 13.XI.1999. U.S.A. California, Riverside, en Citrus spp., 2 hembras, 10.XII.2004 (S. Myartseva, col.).

Comentarios. Nuevo registro para Estados Unidos de América (California) y México de los estados de San Luis Potosí y Tamaulipas.

\section{Eretmocerus naranjae Myartseva, sp. $n$.}

Diagnosis. La hembra de Eretmocerus naranjae sp. n. puede ser distinguida por la maza antenal que es de 5.4 a 6.5 veces tan larga como ancha y de 1.6 a 1.8 veces tan larga como el escapo, el escapo es de 4.3 a 5.3 veces tan largo como ancho, las 6 setas sobre el lóbulo medio del mesoescudo, ovipositor 1.1 a 1.2 veces tan largo como la maza y la tibia media 0.8 a 0.9 veces tan larga como el ovipositor e igual a la maza.

Difiere de las otras especies de Eretmocerus que parasitan a la mosquita blanca $A$. floccosus en América (Rose, 2000) por la relación de la longitud de la maza antenal, el ovipositor y la tibia media: sólo esta especie tiene la maza igual a la tibia media y es más corta que el ovipositor $(0.8$ a $0.9 \mathrm{X})$. E. longiterebrus también tiene la maza subigual a la tibia media pero su ovipositor es 1.26 veces más largo que la maza y la tibia media; en $E$. naranjae es sólo $1.1 \mathrm{X}$ más largo que la maza y la tibia media.

E. naranjae sp. n. es similar a E. comperei y E. gracilis y también a E. corni pero difiere de esas especies en las siguientes características: en E. comperei el segundo segmento funicular es subcuadrado, maza antenal 5.5 - 6.0 veces tan larga como ancha, escapo cerca de 4.3 veces tan largo como ancho, vena marginal del ala anterior 1.6 veces más larga que la vena estigmal, fleco marginal del ala posterior más corto que la anchura máxima del ala; en E. gracilis el segundo segmento funicular es subcuadrado, ala anterior cerca de 2.7 veces tan larga como ancha, vena marginal 1.5 veces tan larga como la vena estigmal, fleco marginal del ala posterior casi igual a la anchura del ala; además, ambas especies tienen una igual longitud de la maza, el ovipositor y la tibia media; en E. corni el segundo segmento funicular es subcuadrado, maza 5.9 - 7.0 veces tan larga como ancha, vena marginal del ala anterior menos de 1.5 veces tan larga como la vena estigmal, ovipositor $0.8 \mathrm{X}$ la longitud de la maza e igual a la tibia media. En E. naranjae el segundo segmento funicular es distintivamente transversal, escapo 4.3-5.3 veces tan largo como ancho, maza 5.4 - 6.5 veces tan larga como ancha, ala anterior 2.4 - 2.5 veces tan larga como ancha, vena marginal cerca de 2 veces tan larga como la vena estigmal, fleco marginal del ala posterior más largo que la anchura máxima del ala; ovipositor 1.1 veces tan largo como la maza y la tibia media, su punta con frecuencia ligeramente curvada.

Descripción. Longitud de especimenes montados en bálsamo de Canadá: 0.75-0.80 mm. Coloración. Cabeza amarilla, cara amarillo claro debajo de los ojos, ojos de color pardo rojizo, mandíbula amarillo oscuro. Cuerpo amarillo, patas amarillo claro, alas hialinas, vena submarginal ligeramente ahumada, ovipositor amarillo oscuro.

Estructura. Cabeza cerca de 1.2 veces tan ancha como alta. Anchura de frontovértice cerca de 0.5 a $0.6 \mathrm{X}$ la anchura de la cabeza. Ocelos posteriores a una distancia de cerca de dos diámetros de un ocelo al margen del ojo. Ojos casi tan largos como las mejillas. Mandíbula con dos dientes y un borde romo corto. Antenas insertadas más cerca del margen de la boca (Fig. 4). Radícula subigual en longitud al pedicelo. Escapo 4.3 a 5.3 veces tan largo como ancho y cerca de $0.6 \mathrm{X}$ la longitud de la maza antenal. Pedicelo 2.5 veces tan largo como ancho y $0.26-0.30 \times$ la longitud de la maza antenal. Primer segmento funicular triangular, segundo segmento distintamente transversal. Maza antenal dorsalmente convexa y 
Myartseva \& Coronado-Blanco: Seis especies de parasitoides de la mosquita blanca

ventralmente casi recta, ápice desde adelgazado hasta truncado; de 5.4 - 6.5 veces tan larga como ancha y 1.6 - 1.8 veces tan larga como el escapo. Lóbulo medio del mesoescudo con 6 setas, axila con una seta, lóbulo lateral con dos setas. Propodeo con una proyección corta medialmente. Ala anterior cerca de 2.4 a 2.5 veces tan larga como ancha, su fleco marginal cerca de $0.3 \mathrm{X}$ la anchura máxima del ala. Vena estigmal cerca de 2 veces más corta que la vena marginal. De 9 a 10 setas entre la vena marginal y la línea calva, base del ala con una seta. Ala posterior cerca de 6.5 veces tan larga como ancha, su fleco marginal más largo que la anchura máxima del ala. Espuela de la tibia media cerca de $0.5 \mathrm{X}$ la longitud del basitarso. Ovipositor expuesto, 1.1 veces tan largo como la maza antenal y la tibia media, su punta con frecuencia ligeramente curvada.

Material examinado. Hembra holotipo. México: Tamaulipas, Jaumave, ex Aleurothrixus floccosus en Citrus limon, 4.X.1998 (S. Myartseva, col.). Paratipos: 1 hembra, mismos datos que holotipo, 2 hembras, 4.IV.2000; Cd. Victoria, ex Aleurothrixus floccosus en Psidium guajava, 2 hembras, 25.XI.1999, 1 hembra, ex Aleurothrixus floccosus en Citrus aurantifolia, 15.II.2003 (S. Myartseva, col.).

\section{Eretmocerus paulistus Hempel}

Eretmocerus paulistus Hempel, 1904: 19.

Descrita de Brasil, especímenes tipo perdidos; un neotipo no designado; existe todavía la posibilidad de que el material de Hempel sea encontrado (Rose, 2000). Redescripciones: Dozier, 1932: 117; Rose 2000: 32 - 34, Figs. 27 - 28.

Hospederos. Aleyrodes herridus Hempel, Aleurothrixus floccosus, Neomaskellia bergii, Bemisia sp.

Distribución. Argentina, Brasil, Chile, Cuba, Haití, Perú, España, Estados Unidos de América (California); México.

Referencias. De Santis, 1979: 337-338; Myartseva y Ruíz Cancino, 2000: 22 (como paulistis). Diagnosis. Lóbulo medio del mesoescudo con 4 setas; maza antenal ampliamente espatulada (Fig. 5), 3.5 veces tan larga como ancha, escapo cerca de 3.5 veces tan largo como ancho, pedicelo cerca de 1.8 veces tan largo como ancho, primer segmento funicular en forma de triángulo, segundo segmento comprimido y transversal; ala anterior cerca de 2.4 veces tan larga como ancha, fleco marginal cerca de $1 / 4$ de la anchura del ala; 9 a 12 setas entre la vena marginal y la línea calva; longitud del espolón de la tibia media/basitarso cerca de 1:1.4; longitud de la maza en relación con el ovipositor y la tibia media 1:1.25, 1:1.05, respectivamente. Comentarios. La presencia de Eretmocerus paulistus en México fue reportada sólo por De Santis (1979). En nuestra opinión es necesario complementar el estudio de los materiales que se han descrito como E. paulistus Hempel.

\section{Eretmocerus portoricensis Dozier}

Eretmocerus portoricensis Dozier, 1932: 115.

Descrita de Puerto Rico, lectotipo hembra en USNM; designada por Rose \& Zolnerowich, 1997: 18; redescrita por Rose, 2000: 34 - 36, Figs. 29 - 31.

Hospederos. Aleurothrixus floccosus, A howardi (Quaintance) (sinónimo de A. floccosus), Tetraleurodes acaciae (Quaintance).

Distribución. República Dominicana, Puerto Rico, Perú, Estados Unidos de América (Florida); México.

Referencias. De Santis, 1989: 66 (México); Alvarado-Mejía \& González-Hernández, 1990: 43. En las siguientes publicaciones: Myartseva \& Ruíz Cancino, 2000: 22, 2005: 473; Myartseva et 
al., 2004: 756; Ruíz Cancino et al., 2005: 934, fue identificado como E. portoricensis, en lugar de Eretmocerus jimenezi.

Diagnosis. Maza antenal con ápice curvado (Fig. 6), fuertemente convexo dorsalmente y recto ventralmente, 3.2-3.8 veces tan larga como ancha, escapo 3.5 veces tan largo como ancho, pedicelo 2.2 veces tan largo como ancho, igual a la radícula, primer segmento funicular triangular, tan ancho como largo, segundo segmento 1.6 veces tan ancho como largo; lóbulo medio del mesoescudo con 6 setas; ala anterior 2.7 veces tan larga como ancha, fleco marginal cerca de $0.2 \times$ la anchura del ala; 10 a 13 setas entre la vena marginal y la línea calva; longitud del espolón de la tibia media/basitarso cerca de 1:1.6; ovipositor 1.2 veces tan largo como la maza, igual en longitud a la tibia media.

Comentarios. Eretmocerus portoricensis fue registrado para México por De Santis (1989) y posteriormente por Alvarado-Mejía y González-Hernández (1990). Posiblemente en México, esta especie también es E. jimenezi Rose porque Rose \& Zolnerowich (1997) y Rose (2000) no reportaron en la distribución a E. portoricensis para México.

\section{AGRADECIMIENTOS}

Las autoras agradecen al Dr. G.A. Evans (USDA/APHIS c/o Systematic Entomology Laboratory, Beltsville, MD, USA) por el envío de artículos importantes sobre Aphelinidae, incluyendo del género Eretmocerus. La primera autora agradece a su colega Dr. D.R. Kasparyan (UAM Agronomía y Ciencias, UAT, Tamaulipas, México and Zoological Institute of Russian Academy of Sciences, Sanct Petersburg, Russia) por su ayuda amistosa a través del período de estudio de Aphelinidae en México. Al Dr. Enrique Ruíz Cancino, por la revisión del manuscrito, así como a los revisores por sus importantes observaciones y sugerencias. A la División de Estudios de Postgrado e Investigación, UAM Agronomía y Ciencias, Universidad Autónoma de Tamaulipas, Ciudad Victoria, Tamaulipas, México por el apoyo financiero a este trabajo.

\section{LITERATURA CITADA}

Alvarado-Mejía, G. \& A. González-Hernández. 1990. Taxonomía de las especies de Aphytis Howard (Hymenoptera: Aphelinidae) y otros géneros en el área citrícola de Nuevo León. Biotam 2 (3): 42-51.

De Santis, L. 1979. Catálogo de los himenópteros calcidoideos de América al Sur de los Estados Unidos. La Plata, Argentina. 488 p. . 1989. Catálogo de los himenópteros calcidoideos (Hymenoptera) al Sur de los Estados Unidos. Segundo Suplemento. Acta Entomológica Chilena 15: 9-90.

Dozier, H. L. 1932. The identity of certain whitefly parasites of the genus Eretmocerus Hald., with descriptions of new species (Hymenoptera: Aphelinidae). Proc. Entomol. Soc. Wash. 34: 112-122.

Ferriere, C. 1965. Hymenoptera Aphelinidae d'Europe et du Bassin Mediterraneen. Masson et Cie Editeurs. Paris. 205 pp.

Haldeman, S. S. 1850. On four new species of Hemiptera of the genera Ploiaria, Chermes and Aleurodes, and two new Hymenoptera, parasitic in the last named genus. Amer. J. Sci. Arts 9: 108-111.

Hayat, M. 1998. Aphelinidae of India (Hymenoptera: Chalcidoidea): a taxonomic revision. Mem. Entomol., Inter. 13, 416 p. 
Myartseva \& Coronado-Blanco: Seis especies de parasitoides de la mosquita blanca

Hempel, A. 1904. Notas sobre dois inimigos da laranjeira, Familia Aleurodidae. Boletim da Agricultura (Sao Paolo) 5: 10-21.

Myartseva, S. N. \& E. Ruíz Cancino. 2000. Annotated checklist of the Aphelinidae (Hymenoptera: Chalcidoidea) of Mexico. Folia Entomol. Mex. 109: 7-33.

Myartseva, S. N., E. Ruíz Cancino \& J. M. Coronado Blanco. 2004. Aphelinidae (Hymenoptera), pp.753-757. In: Llorente Bousquets, J., J.J. Morrone, O. Y. Ordóñez y I. V. Fernández (Eds.). Biodiversidad, Taxonomía y Biogeografía de Artrópodos de México: Hacia una síntesis de su conocimiento. México, UNAM.

Noyes, J. S. 1982. Collecting and preserving chalcid wasps (Hymenoptera: Chalcidoidea). J. Nat. Hist. 16: 315-334.

. 2005. Universal Chalcidoidea Database. World Web electronic publication [http:/ /internt.nhm.ac.uk/jdsml/perth/chalcidoids/ (updated 6.X.2005).

Risbec, J. 1951. Les chalcidoides d'A.O.F. Mem. Inst.Franc.Afrique Noire 13: 7-409.

Rose, M. 2000. Eretmocerus Haldeman (Hymenoptera: Aphelinidae) reared from Aleurothrixus floccosus (Maskell) (Homoptera: Aleyrodidae) in the Americas. Vedalia 7 (1): 3-46.

Rose, M. \& P. De Bach. 1994. The woolly whitefly of citrus, Aleurothrixus floccosus (Homoptera: Aleyrodidae). Vedalia 1 (1): 29-60.

Rose, M. \& G. Zolnerowich. 1997. Eretmocerus Haldeman (Hymenoptera: Aphelinidae) in the United States, with descriptions of new species attacking Bemisia (tabaci complex) (Homoptera: Aleyrodidae). Proc. Entomol. Soc. Wash. 99 (1): 1-27.

Ruíz Cancino, E., J. M. Coronado Blanco \& S. N. Myartseva. 2005. Plagas de cítricos y sus enemigos naturales en el estado de Tamaulipas, México. Entomología Mexicana 4: 931-936.

Recibido: 12 de junio 2006

Aceptado: 8 de diciembre 2006 